\title{
First results from the Cassini radio occultations of the Titan ionosphere
}

Avydas J. Kliore, ${ }^{1}$ Andrew F. Nagy, ${ }^{2}$ Essam A. Marouf, ${ }^{3}$ Richard G. French, ${ }^{4}$ F. Michael Flasar, ${ }^{5}$ Nicole J. Rappaport, ${ }^{1}$ Aseel Anabttawi, ${ }^{1}$ Sami W. Asmar, ${ }^{1}$ Daniel S. Kahann, ${ }^{1}$ Elias Barbinis, ${ }^{1}$ Gene L. Goltz, ${ }^{1}$ Don U. Fleischman, ${ }^{1}$ and David J. Rochblatt ${ }^{1}$

Received 17 December 2007; revised 20 March 2008; accepted 6 May 2008; published 23 September 2008.

[1] The first four sets of radio occultations of the Titan's ionosphere were obtained by the Cassini spacecraft between March 2006 and May 2007. These occultations occurred at middle and high latitudes, at solar zenith angles from about $86^{\circ}$ to $96^{\circ}$. The main ionospheric peak was seen, as expected from modeling and previous observations, near $1200 \mathrm{~km}$, with a density of about $1-3 \times 10^{3} \mathrm{~cm}^{-3}$. A consistent ledge near $1000 \mathrm{~km}$ was also seen, and one of the polar observations found a significant $\left(\sim 3 \times 10^{3} \mathrm{~cm}^{-3}\right)$ layer in the region of 500-600 km. This layer also is seen in other observations with a density varying from about 0.7 to $1.7 \times 10^{3} \mathrm{~cm}^{-3}$, suggesting a variable production source (or sources) for this peak.

Citation: Kliore, A. J., et al. (2008), First results from the Cassini radio occultations of the Titan ionosphere, J. Geophys. Res., 113, A09317, doi:10.1029/2007JA012965.

\section{Introduction}

[2] Prior to 1997, there were no physical measurements of the ionosphere of Titan, although modeling based on atmospheric data then available provided theoretical estimates of its structure and composition [cf. Strobel, 1974; Capone et al., 1976; Whitten et al., 1977; Keller et al., 1992; Toublanc et al., 1995; Fox and Yelle, 1997; Anicich and McEwan, 1997; Nagy and Cravens, 1998]. The publication by Bird et al. [1997] of results derived from an analysis of Voyager data obtained in 1981 provided the first experimental observations, and many more theoretical studies followed [cf. Banaszkiewicz et al., 2000; Cravens et al., 1998, 2005, 2006; Galand et al., 1999; Kabin et al., 1999; Ma et al., 2004, 2006; Nagy et al., 2001]. The arrival of Cassini at Saturn for the first time enabled direct in situ observations of the structure and composition of the Titan ionosphere down to the level of about $950 \mathrm{~km}$ by the INMS, CAPS, and RPWS instruments [cf. Hartle et al., 2006; Szego et al., 2005; Wahlund et al., 2005; Young et al., 2005, Waite et al., 2005].

[3] The design of the Cassini spacecraft's tour of the Saturnian system permitted multiple radio occultations of

\footnotetext{
${ }^{1}$ Jet Propulsion Laboratory, California Institute of Technology, Pasadena, California, USA.

${ }^{2}$ Space Physics Research Laboratory, University of Michigan, Ann Arbor, Michigan, USA.

${ }^{3}$ Department of Electrical Engineering, San Jose State University, San Jose, California, USA. USA.

${ }^{4}$ Astronomy Department, Wellesley College, Wellesley, Massachusetts,

${ }^{5}$ NASA Goddard Space Flight Center, Greenbelt, Maryland, USA.
}

Copyright 2008 by the American Geophysical Union. 0148-0227/08/2007JA012965
Titan's ionosphere and atmosphere to be carried out. Of these, a group of four occultations was incorporated into the primary Cassini mission. These occultations occurred on Titan flybys T12 (20 March 2006), T14 (20 May 2006), T27 (26 March 2007), and T31 (28 May 2007). Of these, the first two probed middle latitudes in the Southern Hemisphere $\left(14.3^{\circ} \mathrm{S}\right.$ and $36.3^{\circ} \mathrm{S}$ for $\mathrm{T} 12$ and $19.7^{\circ} \mathrm{S}$ and $21.3^{\circ} \mathrm{S}$ for T14). The other two occultations made observations in polar and near-polar locations $\left(74.6^{\circ} \mathrm{S}\right.$ and $60.6^{\circ} \mathrm{N}$ for $\mathrm{T} 27$ and $75.4^{\circ} \mathrm{N}$ and $74.0^{\circ} \mathrm{S}$ for T31). All observations occurred near the terminator, with the entry measurements occurring on the dark side (SZA 92.2 ${ }^{\circ}-95.8^{\circ}$ ), and the exits on the sunlit side (SZA $85.7^{\circ}$ to $90^{\circ}$ ). The exit measurements at midlatitudes were also near the dusk terminator.A summary of the observations is presented in Table 1, and Figure 1 shows the associated latitudes and solar zenith angles.

\section{Data Acquisition}

[4] During the occultation period, Cassini transmits three sinusoidal radio signals of $0.94,3.6$, and $13 \mathrm{~cm}$-wavelength (Ka-, X-, and S-band, respectively). They are coherently generated on board Cassini from a common ultrastable crystal oscillator (USO) which has a typical Allan deviation better than about $2 \times 10^{-13}$ over 1 to $100 \mathrm{~s}$ time intervals. The coherency allows accurate measurement of individual signal frequency as well as differential effects. The longest wavelength, S-band, is particularly sensitive to plasma along the radio path, hence is the primary signal for probing tenuous regions of Titan's ionosphere. Differential S/X and $\mathrm{X} / \mathrm{Ka}$ measurements were used to derive the electron density profiles, as they are sensitive only to dispersive media, i.e., the ionosphere and the interplanetary plasma. 
Table 1. Cassini Titan Ionosphere Radio Occultations

\begin{tabular}{|c|c|c|c|c|c|c|c|c|c|c|c|c|c|c|}
\hline Observation & Date & $\begin{array}{c}\text { Latitude }^{\mathrm{a}} \\
\text { (deg) }\end{array}$ & $\begin{array}{l}\mathrm{SZA}^{\mathrm{a}} \\
(\mathrm{deg})\end{array}$ & $\begin{array}{c}\text { Ram } \\
\text { Angle } \\
\text { (deg) }\end{array}$ & $\begin{array}{c}\text { LST } \\
\text { (h) }\end{array}$ & $\begin{array}{c}\text { Dawn/ } \\
\text { Dusk }\end{array}$ & $\begin{array}{c}\text { Main } \\
\text { Altitude } \\
(\mathrm{km})\end{array}$ & $\begin{array}{c}\text { Peak } \\
\text { Density } \\
\left(\mathrm{cm}^{-3}\right)\end{array}$ & $\begin{array}{c}\text { Plasma } \\
\text { Scale Height } \\
(\mathrm{km})\end{array}$ & $\begin{array}{c}\text { Lower } \\
\text { Altitude } \\
(\mathrm{km})\end{array}$ & $\begin{array}{c}\text { Peak } \\
\text { Density } \\
\left(\mathrm{cm}^{-3}\right)\end{array}$ & $\begin{array}{c}\text { Topside } \\
\text { Structure? }\end{array}$ & $\begin{array}{c}\text { SEP } \\
\text { (deg) }\end{array}$ & $\begin{array}{c}\text { Baseline } \\
\text { Sigma } \\
\left(\mathrm{cm}^{-3}\right) \\
\end{array}$ \\
\hline $\mathrm{T} 12 \mathrm{~N}$ & 20 Mar 2006 & -14 & 95.0 & 74 & 5.1 & dawn & 1213 & 1487 & 146 & 584 & 706 & na & 124 & 133 \\
\hline $\mathrm{T} 12 \mathrm{X}$ & 20 Mar 2006 & -36 & 87.5 & 93 & 18 & dusk & 1173 & 1749 & 151 & 948 & 1608 & yes & & 97 \\
\hline $\mathrm{T} 14 \mathrm{~N}$ & 20 May 2006 & -20 & 95.8 & 100 & 5.0 & dawn & 1173 & 1344 & 128 & 876 & 819 & no & 66 & 185 \\
\hline $\mathrm{T} 14 \mathrm{X}$ & 20 May 2006 & -21 & 85.7 & 66 & 18 & dusk & 1280 & 1837 & 141 & 512 & 917 & yes & & 163 \\
\hline $\mathrm{T} 27 \mathrm{~N}$ & 26 Mar 2007 & -75 & 92.2 & 94 & 1.6 & polar & 1253 & 1316 & 116 & 516 & 241 & yes & 134 & 65 \\
\hline $\mathrm{T} 27 \mathrm{X}$ & 26 Mar 2007 & 60.6 & 90.9 & 119 & 7.3 & polar & 1246 & 1358 & 126 & 512 & 164 & yes & & 52 \\
\hline $\mathrm{T} 31 \mathrm{~N}$ & 28 May 2007 & -75 & 92.3 & 90 & 24 & polar & 1238 & 2773 & 70 & 588 & 1201 & yes & 72 & 105 \\
\hline T31X & 28 May 2007 & 74 & 88.2 & 78 & 10.0 & polar & 1200 & 2795 & 209 & 537 & 2881 & yes & & 163 \\
\hline
\end{tabular}

${ }^{\mathrm{a}} \mathrm{At} \mathrm{h}=1200 \mathrm{~km}$, the main peak altitude

[5] The Cassini signals perturbed by Saturn's ionosphere are received and recorded at the ground stations of the NASA/JPL Deep Space network (DSN). To maximize the observation signal-to-noise ratio (SNR), the 70-m diameter DSN stations were used to receive the S- and X-band signals. The Ka-band receiving capability is presently available only at the smaller 34-m diameter Beam-Waveguide DSN stations. Depending on the particular occultation, either one or two of the three DSN complexes (Goldstone, Madrid, and Canberra) were used. Typical free-space SNR achieved is 54,48 , and $42 \mathrm{~dB}-\mathrm{Hz}$ at X-, $\mathrm{Ka}-$, and S-band, respectively. These levels of SNR are unprecedented in radio occultation investigations, and they are instrumental in producing the results reported here, which depend on the precise measurement of the very small effects (of the order of $1 \mathrm{mHz}$ peak at X-Band) introduced by the occultation by the Titan ionosphere.

[6] The DSN stations are equipped with two types of receivers. The Tracking Receiver is a closed-loop system that finds, locks, and tracks the carrier of the Cassini signal and produces Doppler and ranging data as well as demodulates the telemetry. The other receiver, called the Radio Science Receiver (RSR), is an open-loop receiver that is driven by a tuning predictions file generated by the Radio Science group based on the latest navigation solution. The RSR is a digital receiver that has a set of bandwidths that

Location of Cassini observations in Lat and SZA

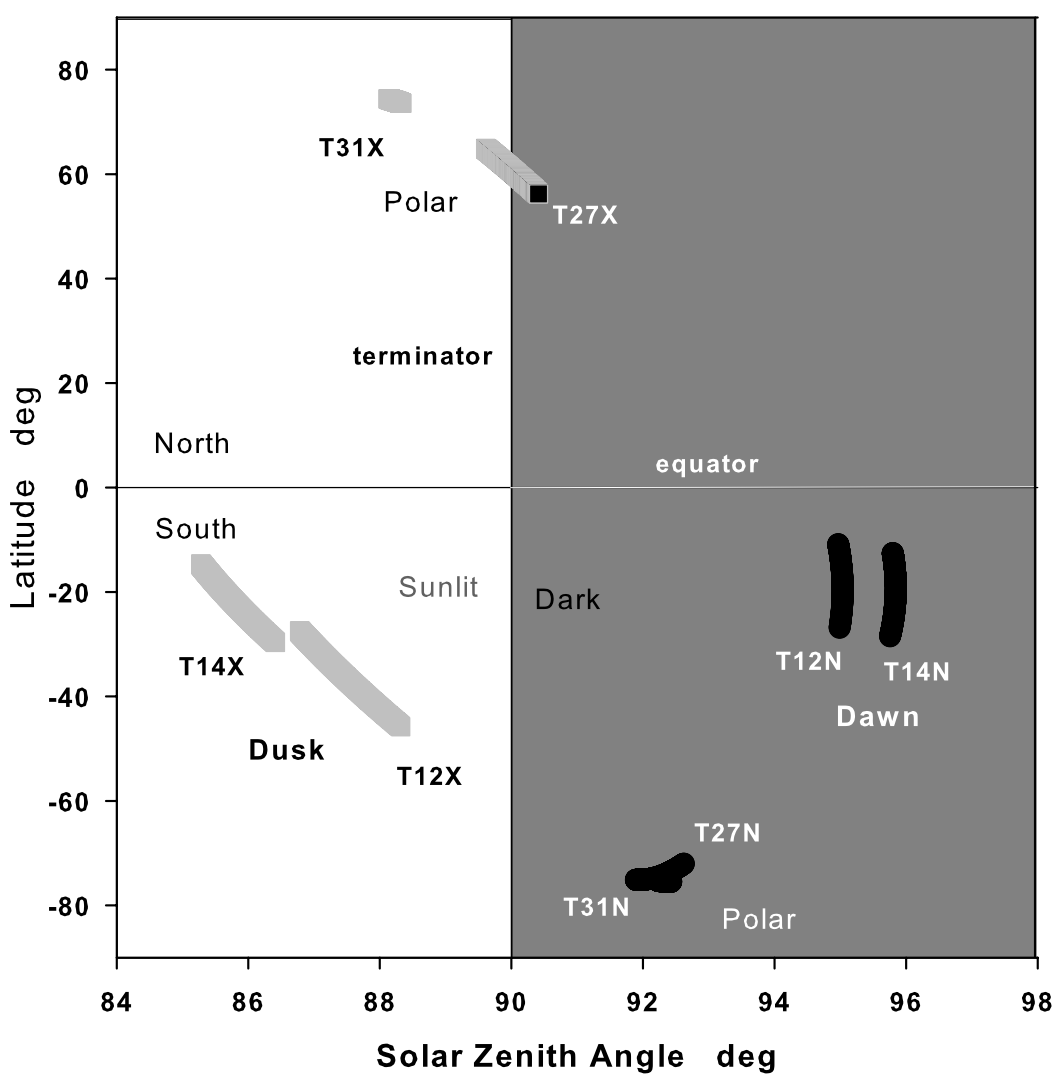

Figure 1. Latitudes and solar zenith angles of the first Cassini radio occultations by the Titan ionosphere ( $\mathrm{X}$ denotes exit and $\mathrm{N}$ denotes entry occultations). 
can be chosen by the user. For a given bandwidth, a matching sampling rate produces complex samples that are delivered to the user at the completion of the station pass.

\section{Data Processing and Analysis}

[7] Digital samples of the in-phase and quadrature signal components were recorded at multiple sampling rates (and bandwidths) using the open-loop Radio Science Receivers (RSR) of the DSN. Analysis results below are based on processing data recorded at $1 \mathrm{kHz}$ bandwidth. The RSR as well as all subsystems of the Deep Space Network are driven by a highly stable frequency and timing system based on a hydrogen maser. Accurate antenna pointing is required especially at higher frequencies (e.g., Ka-band).

[8] The RSR data are processed by first detecting the signal carrier via software. A phase-locked loop or a series of Fast Fourier Transforms are typical detection methods depending on factors such as the signal-to-noise ratio as well as frequency and amplitude dynamics. Once detected, the signal is upconverted to "sky frequency" and then frequency residuals are produced by removing a model of the apparent relative motion between the spacecraft and ground station. These residuals contain the science information on the atmosphere/ionosphere of the planet and form the basic data from which the atmospheric and ionospheric properties are extracted.

[9] The procedures for the analysis and inversion of these data are described in detail elsewhere [cf. Kliore et al., 2004]. The residuals are detrended by fitting a least squares straight line to the baseline data and subtracting it from the raw residuals. The residuals are then used to compute the refractive bending angle at each data time point. This requires a precise ephemeris of Cassini relative to Titan, iterative light propagation time solutions linking the spacecraft, Titan, and the DSN station. The gravitational field of Titan was assumed to be spherical. Once the refractive bending angle and its corresponding ray asymptote distance with respect to the center of refraction are computed for each data point, the Abel integral transform [cf. Kliore et al., 2004], assuming spherical symmetry, is used to invert these data and to produce a vertical profile of refractivity.

[10] Since the radio refractivity is proportional to the electron density times the inverse square of the frequency, the electron density can be determined, as the frequencies are known very precisely. The altitude is determined by height of the closest approach point of the radio line-of-sight relative to the surface of Titan.

\section{Results and Discussion}

[11] The deduced electron density profiles obtained from the four sets of Titan occultations are shown in Figure 2. Each panel gives the profiles for a particular occultation event, with the entry data depicted in black, and the exit in gray. The heavy lines represent averages of data from all observing DSN stations.

[12] In Figure 2 it is apparent that the exit profiles have a higher peak density than the entries. By examining Figure 1 and Table 1 it can be seen that these measurement also fall on the sunlit side of the terminator, and they are located near the dusk terminator.

[13] In discussing dawn and dusk locations one must consider the latitude and the direction of solar illumination. This is shown in the diagram of Figure 3. In order for there to be dusk and dawn, one must be in a location where the Sun is obscured (shadowed) for a substantial portion of the body's rotation period. This obviously occurs at low and midlatitudes. At high latitudes, the summer polar area (in this case the southern one) is always illuminated, while its opposite is dark. During the measurements described here, the solar illumination was from $13^{\circ}$ to $19^{\circ} \mathrm{S}$, and in Figure 3 two sets of shadowing zones are depicted: one which caused by the solid body of Titan, and one which considers both the solid body and Titan's atmosphere and ionosphere (up to the main peak altitude) as the shadowing body. The shadow zones lie in the cross-hatched areas and inward. It can be seen that shadowing by the solid body is not very effective at higher latitude but that atmospheric shadowing, which is appropriate in the context of ionospheric ionization, can extend the dawn dusk effects to higher latitudes.

[14] In Figure 4, the observations are segregated into dawn and dusk groups and averaged. It must be kept in mind, however, that the "dusk" group also is the one that is in sunlight. The "dusk" group has an average main peak density about $50 \%$ greater than the "dawn" group, and also displays more structure above the peak, as well as substantial ionization below the peak. The lower peaks are largest in the unusual profiles from T31.

[15] The error bars in Figures 2 and 4 represent the uncertainties introduced by baseline frequency fluctuations (as they appear in the electron density profiles), and the effects of averaging of several data sets. The baseline fluctuations are caused mostly by plasma in the solar wind, which becomes more important as the radio line-of-sight approached closer to the sun. This is apparent from the last two columns of Table 1, which lists the SEP (Sun-Earthprobe) angle for each observation, and the observed magnitude of the standard deviation of the baseline fluctuation. This is obtained by averaging the electron density points over a portion of the baseline (generally between 1900 and $3000 \mathrm{~km}$ ) to obtain the baseline average $\bar{n}_{e_{b j}}$. For the $j$ th data set, the baseline sigma, $\sigma_{b j}$, which defines the standard deviation of the data, is then given by:

$$
\sigma_{b j}=\frac{\sqrt{\sum_{i}\left(n_{e i j}-\bar{n}_{e_{b j}}\right)^{2}}}{\sqrt{B-1}}
$$

where $B$ is the number of baseline data points.

[16] Data for each observation are collected by multiple DSN stations, ranging from two to five; therefore data sets must be averaged to obtain one electron density profile for each observation. So, if there are $\mathrm{N}$ data sets, the standard deviation of the average for each point $i$ will be

$$
\sigma_{A_{i}}=\frac{\sqrt{\sum_{j}\left(n_{e_{i j}}-\bar{n}_{e_{i}}\right)^{2}}}{\sqrt{(N-1) N}}
$$



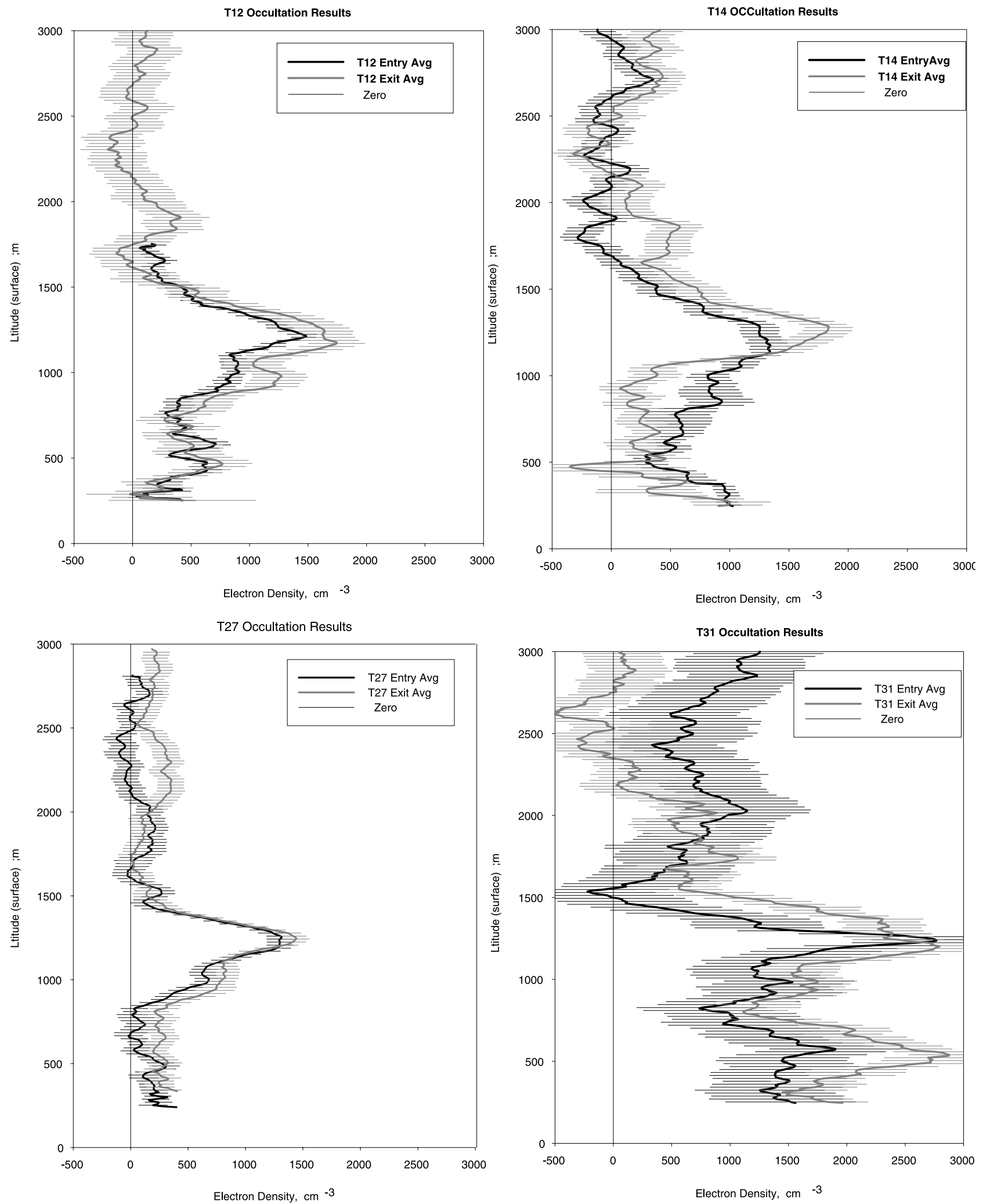

Figure 2. Averaged electron density profiles from the T12, T14, T27, and T31 Titan radio occultations. The entry measurements are shown in black, and the exits are shown in gray. 


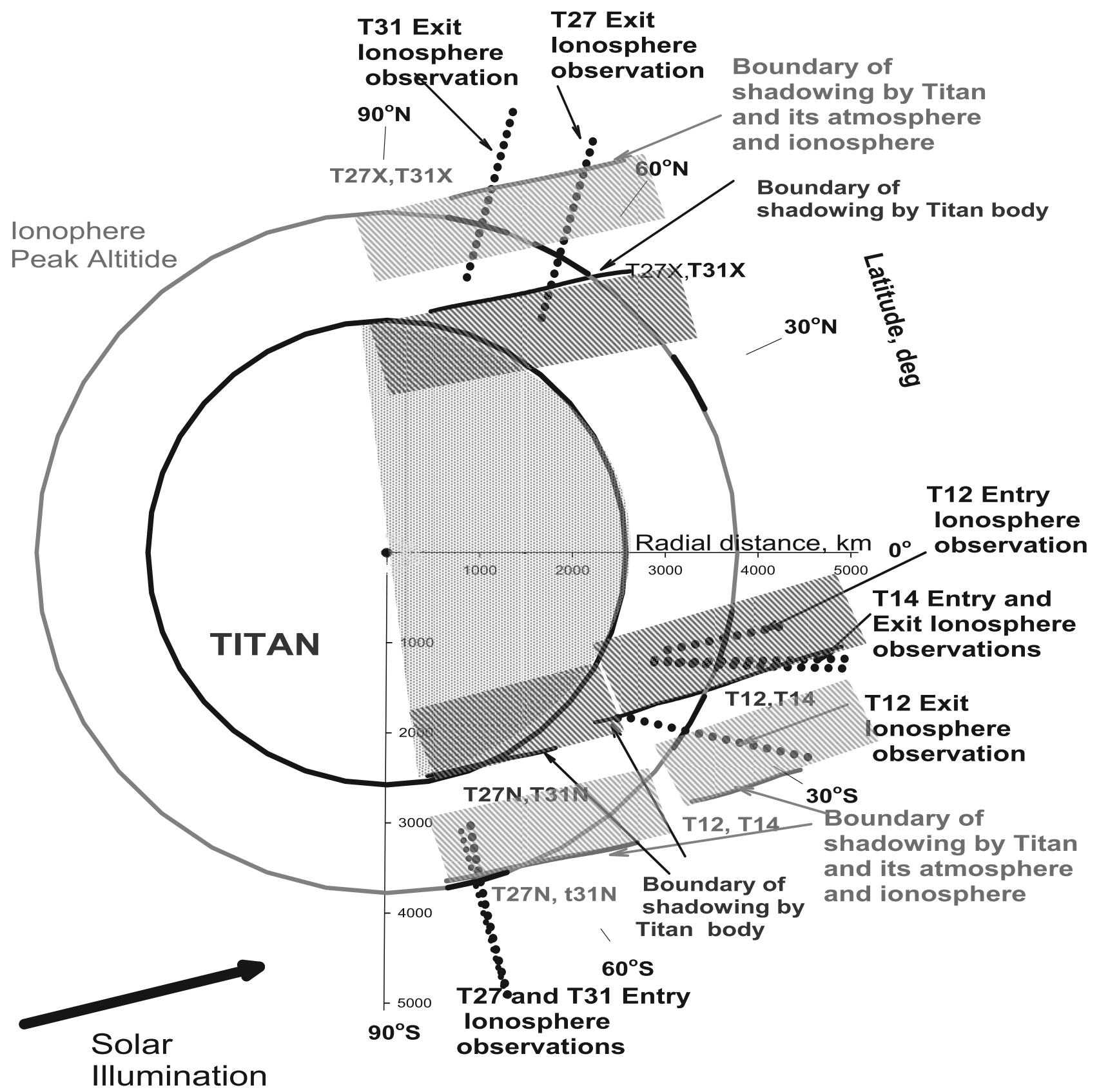

Figure 3. Solar shadowing zones by the Titan body only and by the body and atmosphere of Titan. The zones defined by the cross-hatched areas and inward represent shadowing during each Titan revolution.

where $\bar{n}_{e_{i}}$ is the average for point $i$ from the $\mathrm{N}$ data sets. However, the baseline standard deviation must also be taken into account, and it propagates as

$$
\sigma_{B_{i}}=\frac{1}{N} \sqrt{\sum_{j} \sigma_{b j}^{2}}
$$

The total standard deviation for point $i$ of the $k$ th averaged observation is

$$
\sigma_{k i}=\sqrt{\sigma_{A_{i}}^{2}+\sigma_{B_{i}}^{2}}
$$

This is the quantity that is plotted as the error bars in Figures 2 and 4 . These are quite different from one observation to another, reflecting the effect of the SEP angle, with the largest error bars occurring for those observations nearest to solar conjunction (T14 and T31).

[17] When averaging the dusk and the dawn observations, it is desirable to weight the more accurate observations higher than the less accurate ones. For that purpose, a weighting factor is defined as follows:

$$
w_{k i}=\frac{1}{\sigma_{k i}^{2}}
$$




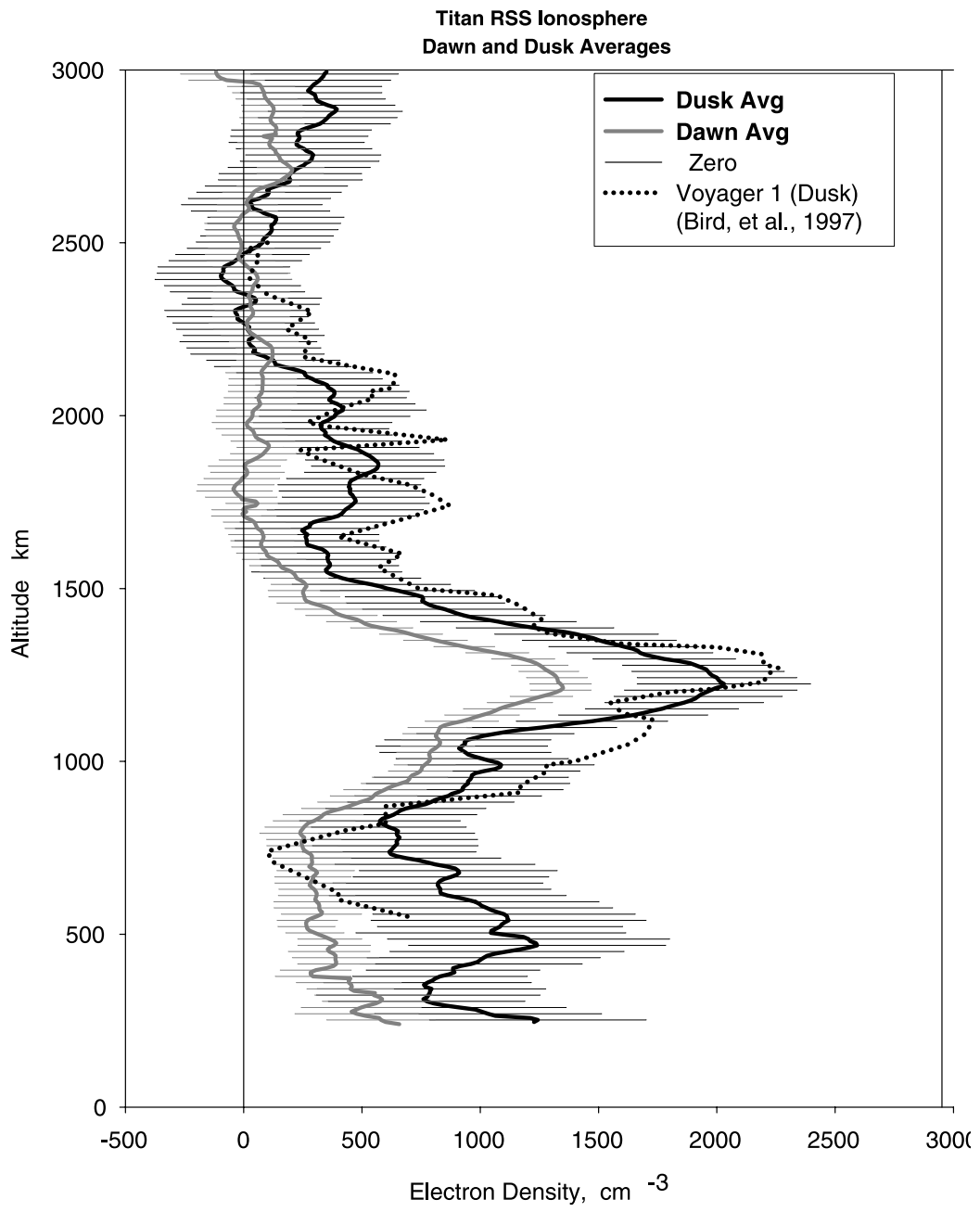

Figure 4. Observed dawn and dusk and dawn averaged electron density profiles. For T12 and T14 (southern midlatitudes), the dusk observations are also in sunlight, and the dawn observations are in the dark (see Figure 1). The Voyager 1 profile is also shown for comparison.

It is convenient to normalize the weighting factors so that their sum is equal to the number of observations being averaged, $K$. Then, the normalized weighting factors become

$$
w_{N_{k i}}=\frac{K w_{k i}}{\sum_{k} w_{k i}}
$$

The weighted average for point $i$ is then

$$
\bar{n}_{e_{i}}=\frac{1}{K} \sum_{k} w_{N_{k i}} n_{e_{k i}}
$$

and the standard deviation of the weighted average is

$$
\sigma_{a v_{i}}=\sqrt{\frac{\sum_{k}\left(\bar{n}_{e i}-w_{N_{k i}} n_{e_{k i}}\right)^{2}}{K(K-1)}}
$$

The error standard deviation carried forward from each data set propagates to produce

$$
\sigma_{p_{i}}=\frac{1}{K} \sqrt{\sum_{k}\left(w_{N_{k i}} \sigma_{k i}\right)^{2}}
$$

And finally, since the two errors described by equations (8) and (9) can be independent, the total one-sigma uncertainty of the weighted average is

$$
\sigma_{i}=\sqrt{\sigma_{a v_{i}}^{2}+\sigma_{p_{i}}^{2}}
$$

This is the quantity that is plotted in the error bars on the averaged plots of Figure 4. The uncertainty in altitude is determined by the uncertainty in the projection of the position of the spacecraft relative to Titan, which at the times of these measurements was less than $1 \mathrm{~km}$ (one-sigma).

[18] The averaged profiles provide several puzzling comparisons. One of those is the great difference between the 


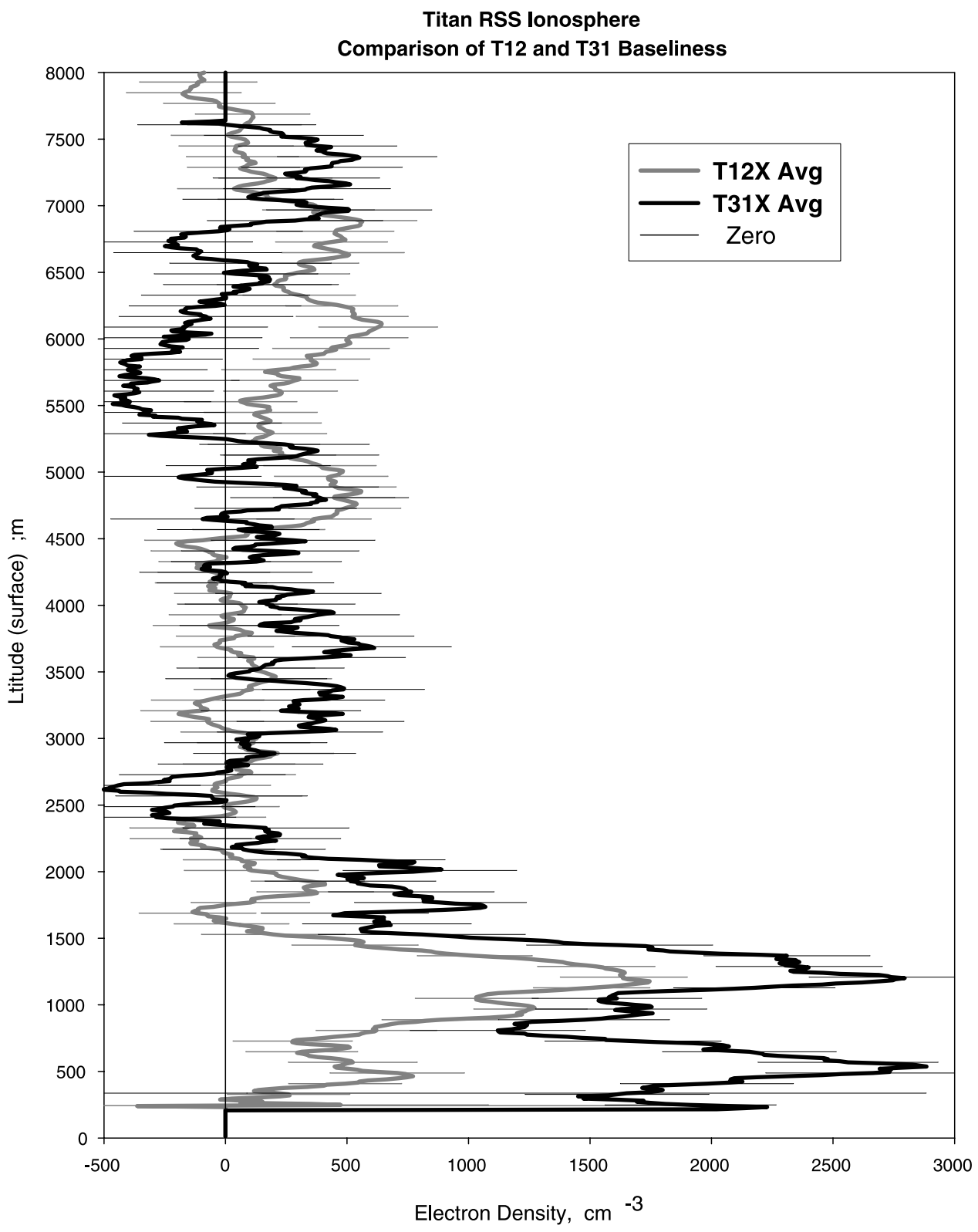

Figure 5. A comparison of $\mathrm{T} 12 \mathrm{X}$ and $\mathrm{T} 31 \mathrm{X}$ long baselines. The baseline fluctuations are similar, indicating that interplanetary plasma fluctuations are not responsible for the large differences in the ionosphere profiles.

profiles of T27 and those from T31. Although both were polar measurements, taken at very similar solar illumination conditions (see Table 1), the resulting profiles are dramatically different. This could be caused by either a sudden and major change in interplanetary plasma fluctuations or that the observed is real, which we believe is the likely scenario (see later discussion associated with Figure 5).

[19] In Figure 5 two of the measurements having long baselines, $\mathrm{T} 12 \mathrm{X}$, and $\mathrm{T} 31 \mathrm{X}$, are plotted. No obvious increase in baseline fluctuations is seen in the data from T31. Therefore it may be assumed that the electron density profiles of T31, which exhibit very substantial lower peaks at about 500-700 km altitude, are real ionospheric structures. The T31 observations also differ from the others in their topside plasma scale heights, with the one for the entry being much smaller, and the exit one much greater than the others (see Table 1).

[20] The previous radio occultation observation by Voyager [Bird et al., 1997] and pre-Cassini model calculations did indicate the presence of the main peak at about $1200 \mathrm{~km}$ altitude which appears in all of the Cassini radio occultation measurements. The models were successful of reproducing the observed altitude and magnitude of this peak, based solely on ionization by solar EUV radiation and the resulting photoelectrons [e.g., Fox and Yelle, 1997; Keller et al., 1998]. The Cassini Langmuir probe [Wahlund et al., 2005] and ion mass spectrometer [Cravens et al., 2005] results also found similar electron densities, although the presence of the peak was not always clearly seen, most likely because of the nature of the orbits. The comprehensive MHD model 


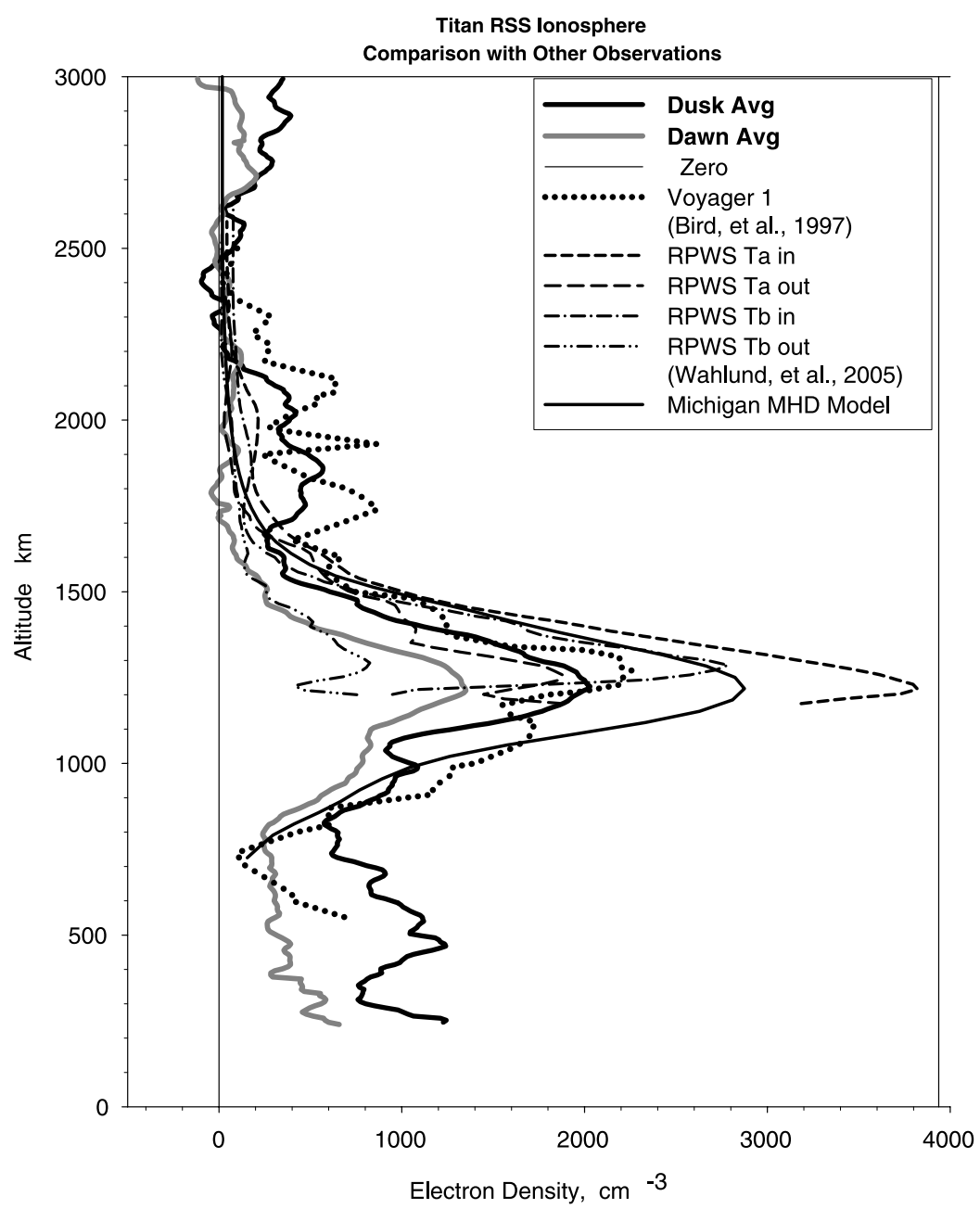

Figure 6. Comparison with other observations and modeling.

results of Ma et al. [2006] demonstrated unambiguously that chemical equilibrium conditions hold below about $1400 \mathrm{~km}$, which are important to keep in mind for the discussion that follow. Thus the processes controlling the height and magnitude of this main peak is reasonably well understood at this time. The electron density profiles from the Voyager occultation, the Cassini Langmuir probe, and the Michigan MHD model are coplotted with our dawn and dusk averages in Figure 6.

[21] The shoulder in the electron densities below the main peak ( $\sim 1000 \mathrm{~km}$. altitude) is most likely the result of a similar shoulder in the photoionization production rate. The solar production rates calculated by Cravens et al. [2005] show such a shoulder, which is the result of solar soft $\mathrm{X}$ rays. These X-ray fluxes are known to be quite variable in both intensity and time [e.g., Lean et al., 2003], consistent with the observed changing densities in this $800-1100 \mathrm{~km}$ chemical equilibrium region.

[22] In a very recent paper, Cravens et al. [2008] show that ion precipitation, consistent with Cassini observations, will result in significant electron-ion production rates, in the $500-700 \mathrm{~km}$ region. Here again, given that chemical equilibrium conditions prevail, production rates are directly proportional to electron densities. The energy and compo- sition of the precipitating particles determines the deposition height and production efficiency, while the ion chemistry controls the effective recombination rates. Therefore, the task of calculating quantitative electron density values is an extremely difficult one at this time, given all the uncertainties in these parameters, and only order of magnitude estimates are feasible; the range of peak densities suggested by Cravens et al. [2008] is $0.4-1.6 \times 10^{3} \mathrm{~cm}^{-3}$. Energetic electron precipitation is also a possible source of ionization at these altitudes, although given the small gyroradii of precipitating electrons, there must be "magnetic connection" to the low-altitude ionosphere from the region(s) where such electrons have been observed.

[23] It was also suggested by Molina-Cuberos et al. [2001] that meteoric impact will cause low altitude ionization at Titan. These authors recently published the results of their model calculations, which considered metallic ion chemistry resulting from the ablation of meteoroids and the creation of long-lived metallic ions. They predict electron densities ranging from $10^{3}$ to $10^{4} \mathrm{~cm}^{-3}$ in the altitude range of $600-800 \mathrm{~km}$. Thus, this process, which also causes sporadic layers in Earths ionosphere, can also fully or partially account for the electron densities observed during T31. 
[24] The Cassini Ion-Neutral Mass Spectrometer (INMS) has observed significant wave structure in the neutral atmosphere in 1200-1400 km region [Waite et al., 2005; Müller-Wodarg et al., 2006]. In this altitude region, presumably controlled by chemical equilibrium, the ion densities follow directly the changes in the neutral densities. Thus if the wave like features seen in the electron densities in this region above the main density peak is real and not an indication of noise in the data, it can be accounted for by the presence of the wave structure in the neutral density.

[25] Finally, we need to address the dusk to dawn drop in the average observed electron densities. The average solar zenith angle of the dusk measurements is about $88^{\circ}$ (see Table 1). Thus these dusk densities are mainly caused by photoionization and photoelectrons, with a significantly smaller contribution due to impact ionization by magnetospheric electrons and ions [cf. Agren et al., 2007]. This was demonstrated by quantitative model calculations, as mentioned earlier [e.g., Cravens et al., 2005; Keller et al., 1998]. On the other hand, by dawn the relative importance of solar radiation versus magnetospheric electrons is reversed. The average solar zenith angle of the dawn observations is about $94^{\circ}$. Given the relatively long time that Titan's ionosphere is without sunlight, recombination removes a significant fraction of the ionization from the previous day. The unit optical depth for EUV radiation is at about $1250 \mathrm{~km}$ for $90^{\circ}$ solar zenith angle [Cravens et al., 2005]; thus the effective occulting disk has a radius of about $3825 \mathrm{~km}$. This means that the observed dawn densities are mainly due to direct impact ionization by magnetospheric electrons. The various ionospheric models [e.g., Ma et al., 2004; Cravens et al., 2005] do indicate qualitatively that magnetospheric electron impact ionization leads to lower densities, consistent with this observed behavior.

[26] Acknowledgments. The work described in this paper was supported by the Cassini Program at JPL. One of the authors, AFN, was also partially supported in this work by NASA grant NAG5-13332. The authors wish to express their gratitude to the personnel of the Deep Space Net (DSN), who were instrumental in the successful acquisition of their data.

[27] Zuyin Pu thanks Jan-Erik Wahlund and Jack Waite for their assistance in evaluating this paper.

\section{References}

Agren, K., et al. (2007), On magnetospheric electron impact ionisation and dynamics in Titan's ram-side and polar ionosphere - a Cassini case study, Ann. Geophys., 25(11), 2359-2369.

Anicich, V. G., and M. J. McEwan (1997), Ion-molecule chemistry in Titan's ionosphere, Planet. Space Sci., 45(8), 897-921, doi:10.1016/ S0032-0633(97)00053-6.

Banaszkiewicz, M., et al. (2000), The upper atmosphere and ionosphere of Titan: A coupled model, in Planetary Ionospheres and Magnetospheres, edited by T. E. Cravens, pp. 1547-1550, Pergamon, New York.

Bird, M. K., et al. (1997), Detection of Titan's ionosphere from Voyager 1 radio occultation observations, Icarus, 130(2), 426-436, doi:10.1006/ icar.1997.5831.

Capone, L. A., et al. (1976), Lower ionosphere of Titan, Icarus, 28(3), 367-378, doi:10.1016/0019-1035(76)90150-0.

Cravens, T. E., C. J. Lindgren, and S. A. Ledvina (1998), A two-dimensional multifluid MHD model of Titan's plasma environment, Planet. Space Sci., 46(9-10), 1193, doi:10.1016/S0032-0633(98)00051-8.

Cravens, T. E., et al. (2005), Titan's ionosphere: Model comparison with Cassini Ta data, Geophys. Res. Lett., 32, L12108, doi:10.1029/ 2005 GL023249.
Cravens, T. E., et al. (2006), Composition of titan's ionosphere, Geophys. Res. Lett., 33, L07105, doi:10.1029/2005GL025575.

Cravens, T. E., et al. (2008), Energetic ion precipitation at Titan, Geophys. Res. Lett., 35, L03103, doi:10.1029/2007GL032451.

Fox, J. L., and R. V. Yelle (1997), Hydrocarbon ions in the ionosphere of Titan, Geophys. Res. Lett., 24, 2179-2182, doi:10.1029/97GL02051.

Galand, M., J. Lilensten, D. Toublanc, and S. Maurice (1999), The ionosphere of Titan: Ideal diurnal and nocturnal cases, Icarus, 140(1), $92-$ 105, doi:10.1006/icar.1999.6113.

Hartle, R. S., et al. (2006), Initial interpretation of Titan plasma interaction as observed by the Cassini plasma spectrometer: Comparisons with Voyager 1, Planet. Space Sci., 54(12), 1211-1224, doi:10.1016/j.pss. 2006.05.029.

Kabin, K., et al. (1999), Interaction of the Saturnian magnetosphere with Titan: Results of a three-dimensional MHD simulation, J. Geophys. Res., 104(A2), 2451-2458, doi:10.1029/1998JA900080.

Keller, C. N., T. E. Cravens, and L. Gan (1992), A Model of the Ionosphere of Titan, J. Geophys. Res., 97(A8), 12,117-12,135, doi:10.1029/92JA00231.

Keller, C. N., et al. (1998), Model of Titan's ionosphere with detailed hydrocarbon chemistry, Planet. Space Sci., 46, 1157-1174, doi:10.1016/S0032-0633(98)00053-1.

Kliore, A. J., et al. (2004), Cassini Radio Science, Space Sci. Rev., 115, 1-70, doi:10.1007/s11214-004-1436-y.

Lean, J. L., et al. (2003), A new model of solar EUV irradiance variability 2. Comparisons with empirical models and observations and implications for space weather, J. Geophys. Res., 108(A2), 1059, doi:10.1029/ 2001JA009238.

Ma, Y. J., et al. (2004), 3-D global MHD model prediction for the first close flyby of Titan by Cassini, Geophys. Res. Lett., 31(22), L22803, doi:10.1029/2004GL021215

Ma, Y., et al. (2006), Comparisons between MHD model calculations and observations of Cassini flybys of Titan, J. Geophys. Res., 111, A05207, doi:10.1029/2005JA011481.

Molina-Cuberos, G. J., et al. (2001), Ionosphere layer induced by meteoric ionization in Titan's atmosphere, Planet. Space Sci., 49, 143-153, doi:10.1016/S0032-0633(00)00133-1.

Müller-Wodarg, I. C. F., et al. (2006), Waves and horizontal structure in Titan's thermosphere, J. Geophys. Res., 111, A12315, doi:10.1029/ 2006JA011961

Nagy, A. F., and T. E. Cravens (1998), Titan's ionosphere: A review, Planet. Space Sci., 46(9-10), 1149-1155, doi:10.1016/S0032-0633(98)00049-X.

Nagy, A. F., et al. (2001), The interaction between the magnetosphere of Saturn and Titan's ionosphere, J. Geophys. Res., 106(A4), 6151-6160, doi:10.1029/2000JA000183.

Strobel, D. F. (1974), Photochemistry of hydrocarbons in the atmosphere of Titan, Icarus, 21(4), 466-470, doi:10.1016/0019-1035(74)90149-3.

Szego, K., et al. (2005), The global plasma environment of Titan as observed by Cassini Plasma Spectrometer during the first two close encounters with Titan, Geophys. Res. Lett., 32, L20S05, doi:10.1029/2005GL022646.

Toublanc, D., et al. (1995), Photochemical modeling of Titan's atmosphere, Icarus, 113(1), 2-26, doi:10.1006/icar.1995.1002.

Wahlund, J.-E., et al. (2005), Cassini measurements of cold plasma in the ionosphere of Titan, Science, 308, 986-989, doi:10.1126/science. 1109807.

Waite, J. H., Jr., et al. (2005), Ion neutral mass spectrometer results from the first flyby of Titan, Science, 308, 982-986, doi:10.1126/science. 1110652.

Whitten, R. C., L. A. Capone, L. McCulley, and P. F. Michelson (1977), Upper Ionosphere of Titan, Icarus, 31(1), 89-96, doi:10.1016/0019-1035(77) 90072-0.

Young, D. T., et al. (2005), Composition and dynamics of plasma in Saturn's magnetosphere, Science, 307, 1262 -1266, doi:10.1126/science.1106151.

A. Anabttawi, S. W. Asmar, E. Barbinis, D. U. Fleischman, G. L. Goltz, D. S. Kahann, A. J. Kliore, N. J. Rappaport, and D. J. Rochblatt, Jet Propulsion Laboratory, California Institute of Technology, Pasadena, CA 91109, USA. (akliore@jpl.nasa.gov)

F. M. Flasar, NASA Goddard Space Flight Center, Greenbelt, MD 20771, USA.

R. G. French, Astronomy Department, Wellesley College, Wellesley, MA 02481, USA.

E. A. Marouf, Department of Electrical Engineering, San Jose State University, San Jose, CA 95192, USA.

A. F. Nagy, Space Physics Research Laboratory, University of Michigan, Ann Arbor, MI 48109, USA. 